\section{Antiproliferative Effects Associated with Membrane Lipid Interaction of Green Tea Catechins}

\author{
Hironori Tsuchiya, ${ }^{*}, a$ Toshiyuki Tanaka, ${ }^{b}$ \\ and Motohiko Nagayama ${ }^{a}$
}

${ }^{a}$ Department of Dental Basic Education, Asahi University School of Dentistry, 1851 Hozumi, Mizuho, Gifu 501-0296, Japan and ${ }^{b}$ Laboratory of Medicinal Resources, Gifu Pharmaceutical University, 5-6-1 Mitahora-higashi, Gifu 502-8585, Japan

(Received April 12, 2008; Accepted June 10, 2008; Published online June 20, 2008)

In the light of a novel mode of action, the antiproliferative effects of green tea catechins were studied with relating to their membrane lipid interactions. Mouse myeloma cells and liposomes consisting of phospholipids and cholesterol were treated with structurally-different catechins of 10 and $100 \mu \mathrm{M}$ for $0.5-48 \mathrm{hr}$. The induced changes in membrane fluidity were comparatively determined by measuring fluorescence polarization with different probes to characterize the membrane-acting sites. (-)-Epicatechin3-gallate (ECG) and (-)-epigallocatechin-3-gallate (EGCG) showed the growth-inhibitory effects on tumor cells with the potency increasing in this order, but neither (-)-epicatechin (EC) nor (+)-catechin (C). Simultaneously with inhibiting the cell growth, both catechin gallates rigidified tumor cell membranes by acting on their hydrophilic and hydrophobic regions. The most antiproliferative EGCG predominantly affected the centers of cell membranes and its acting site was deeper with increasing the culture time. Correlating to the comparative effects on tumor cells, EGCG reduced the fluidity of liposomal membranes more intensively than ECG, whereas EC and $\mathrm{C}$ were essentially ineffective. The antiproliferative effects of green tea catechins are associated with their structure-dependent interactions with lipid bilayers to modify cell membrane fluidity.

Key words — green tea, catechin, antiproliferative ef-

*To whom correspondence should be addressed: Department of Dental Basic Education, Asahi University School of Dentistry, 1851 Hozumi, Mizuho, Gifu 501-0296, Japan. Tel.: +81-58329-1266; Fax: +81-58-329-1266; E-mail: hiro@dent.asahiu.ac.jp fect, cell membrane fluidity, membrane lipid interaction

\section{INTRODUCTION}

Beyond the value as a beverage, green tea possesses medicinal and health-supporting functions such as cytoprotective, antioxidant, antiplatelet, antibacterial, antiallergic, anti-inflammatory, hypolipidemic activity, etc. ${ }^{1)}$ In particular, increasing attention has been paid to the cancer prevention and therapy by green tea which affects diverse cellular events relating to cancer development. These effects are attributed to polyhydroxyflavan-3-ol or catechin and its derivatives, the constituents in tea leaves (Camellia sinensis), which inhibit proliferation, metastasis and lipid peroxidation of tumor cells. In addition to conventional mechanisms, another mode of action on biomembranes has been implicated in antiproliferative medicines and food components. ${ }^{2)}$ Cell membranes, as well as membranous organelles, are regarded as one of novel targets for antitumor agents. ${ }^{3)}$ The lipid environments of biomembranes modulate the activities of enzymes and receptors embedded in them. While many tumor cell lines show different membrane properties, like fluidity changes, from their normal counterparts, ${ }^{4)}$ the fluidity modification of cellular, mitochondrial and liposomal membranes results in the growth inhibition of tumor cells. ${ }^{5,6)}$ Membrane fluidity also affects enzymes and lipid hydroperoxides responsible for carcinogenesis. ${ }^{7)}$

Green tea catechins consist of stereoisomers and 3 -gallate esters. Their structure-activity relationship indicates that (+)-catechin (C), (-)-epicatechin (EC), (-)-epicatechin-3-gallate (ECG) and (-)epigallocatechin-3-gallate (EGCG) are suitable for the comparative study. They are not only discriminable in antitumor and membrane activity, ${ }^{8)}$ but also quantitatively the representative constituents in green tea. ${ }^{9)}$ The purpose of this study was to compare the antiproliferative effects of C, EC, ECG and EGCG with relating to their effects on cell membranes and lipid bilayers to modify the membrane fluidity.

\section{MATERIALS AND METHODS}

Materials $-\mathrm{C}, \mathrm{EC}, \mathrm{ECG}$ and EGCG purified from tea leaves were obtained from Kurita (Tokyo, Japan). Their purity was confirmed to be more than 
99\% by HPLC. ${ }^{10)}$ Phospholipids: 1-palmitoyl-2oleoylphosphatidylcholine (POPC), 1-palmitoyl2-oleoylphosphatidylethanolamine (POPE) and 1-stearoyl-2-oleoylphosphatidylserine (SOPS) were purchased from Avanti Polar Lipids (Alabaster, AL, U.S.A.), and cholesterol from Wako Pure Chemicals (Osaka, Japan). Fluorescent probes: 1-anilinonaphthalene-8-sulfonic acid (ANS), $N$-phenyl-1-naphthylamine (PNA), 2-(9-anthroyloxy)stearic acid (2-AS), 6-(9-anthroyloxy)stearic acid (6-AS), 9-(9-anthroyloxy)stearic acid (9-AS), 12-(9-anthroyloxy)stearic acid (12-AS) and 16-(9anthroyloxy)palmitic acid (16-AP) were obtained from Molecular Probes (Eugene, OR, U.S.A.). Dimethyl sulfoxide (DMSO) of spectroscopic grade (Kishida, Osaka, Japan) was used for preparing sample solutions. All other reagents were of the highest grade available commercially.

Cell Culture — Mouse myeloma cells (Sp2/OAg14) were maintained in Dulbecco's modified Eagle's medium supplemented with $10 \%$ (v/v) fetal calf serum (ICN Biomedicals, Aurora, OH, U.S.A.) at $37^{\circ} \mathrm{C}$ in a humidified $5 \% \mathrm{CO}_{2}$ atmosphere. This cell line was used because it was suitable for membrane fluidity experiments, in which the cells were readily suspended in phosphate-buffered saline of pH 7.4 (PBS) and successfully labeled with different probes.

Antiproliferative Effect — The antiproliferative effects of catechins were assayed as reported previously. ${ }^{11)}$ In brief, the cells were inoculated at $3.0 \times 10^{5}$ cells $/ \mathrm{ml}$ in culture medium, to which the DMSO solutions of C, EC, ECG and EGCG were added to be $100 \mu \mathrm{M}$ for each. The final concentration of DMSO was $0.5 \%(\mathrm{v} / \mathrm{v})$ in medium. After culture for 24 and $48 \mathrm{hr}$, the number of viable cells was compared with controls to obtain cell growth inhibition (\%).

Cell Membrane Fluidity Change — The cells were cultured with or without C, EC, ECG and EGCG $(100 \mu \mathrm{M}$ for each) as described above. An aliquot $(2 \mathrm{ml})$ of cell cultures was collected after culture for $1-48 \mathrm{hr}$. After centrifugation, the collected cells were washed twice with and suspended in PBS at $5.0 \times 10^{4}$ cells $/ \mathrm{ml}$. They were labeled with ANS, PNA, 2-AS, 6-AS, 9-AS, 12-AS or 16$\mathrm{AP}$, and then the fluidity changes of cell membranes were determined by measuring fluorescence polarization as reported previously. ${ }^{8}{ }^{8}$ 12) Compared with controls (treated with DMSO vehicle), the increases of polarization values mean the reduction of membrane fluidity, membrane rigidification. ANS and
PNA indicate the fluidity changes in hydrophilic and hydrophobic upper regions of membranes, respectively. Since $n$-(9-anthroyloxy) fatty acids $(n=2$, $6,9,12,16)$ selectively locate at a graded series of levels in lipid bilayers, fluorescence polarization reflects the fluidity gradient extending from the surface to the center of membranes with an increase of $n$. In order to characterize the membrane-acting sites of catechins, their induced changes relative to control polarization values (\%) were compared between $n$-AS(P) polarization. ${ }^{13)}$

Membrane Lipid Interaction — Liposomal tumor cell model membranes of the lipid bilayer structure were prepared as the suspensions in PBS by the previous method. ${ }^{14)}$ The membrane lipid composition was POPC, POPE, SOPS and cholesterol (48:24:8:20, mol\%; total lipids of $0.14 \mathrm{mM}$ ), corresponding to that of tumor cell membranes. ${ }^{15)}$ The DMSO solutions of C, EC, ECG and EGCG were added to the membrane preparations to give a final concentration of $10 \mu \mathrm{M}$ for each, followed by incubation at $37^{\circ} \mathrm{C}$ for $30 \mathrm{~min}$. The concentration of DMSO was adjusted to be less than $0.5 \%(\mathrm{v} / \mathrm{v})$ in the total volume so as not to influence the membrane fluidity of liposomes. After labeling the liposomes with $n$-AS(P), fluorescence polarization was measured to determine the fluidity changes of liposomal membranes.

Data Analysis — Results are expressed as mean \pm S.E. $(n=5)$. Data were statistically analyzed by Student's $t$-test (StatView 5.0; SAS Institute, Cary, NC, U.S.A.). $p$-Values below 0.05 were considered significant.

\section{RESULTS AND DISCUSSION}

EGCG and ECG showed antiproliferative effects at $100 \mu \mathrm{M}$ with the potency being EGCG $>$ ECG, but neither EC nor C. Compared with control cultures, the growth inhibition of tumor cells cultured for 24 and $48 \mathrm{hr}$ was $70.2 \pm 1.8 \%$ and $85.6 \pm 1.0 \%$ by EGCG, and $34.8 \pm 2.2 \%$ and $69.2 \pm 1.1 \%$ by ECG, respectively. The obtained rank order of antiproliferative activity is the same as that of previous studies, although the tested tumor cells are different. ${ }^{16,17)}$ The comparative growthinhibitory effects on various cell lines suggest the possibility for catechins to act on the cell structures common to tumor cells.

Simultaneously with inhibiting the cell growth, EGCG and ECG rigidified tumor cell membranes 


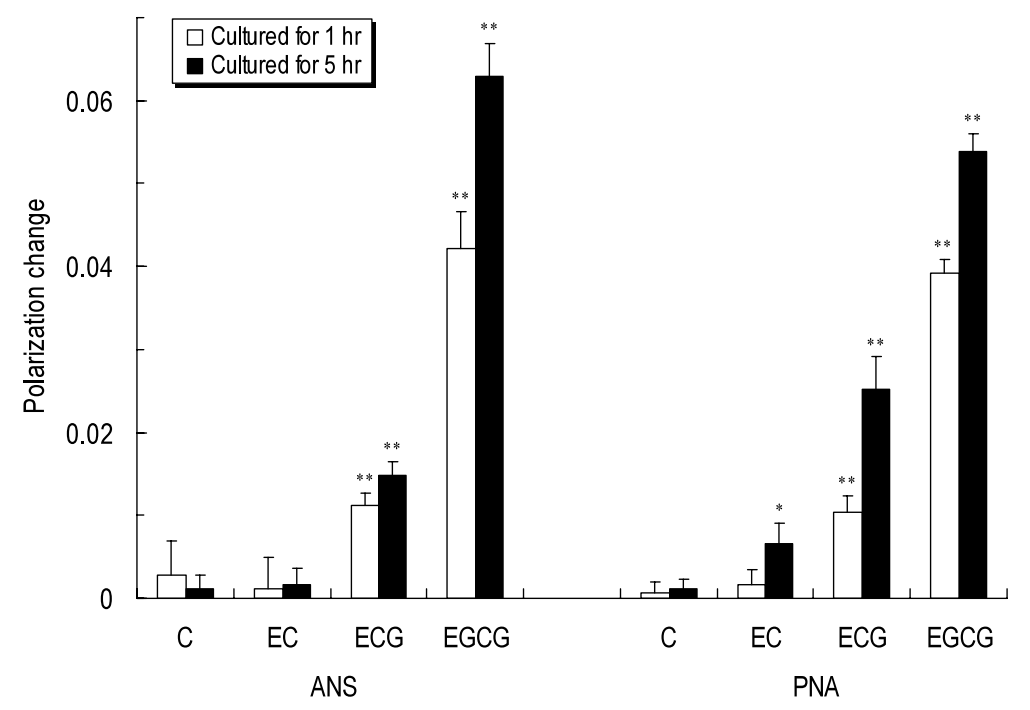

Fig. 1. Effects of Catechins on Cell Membranes

Mouse myeloma cells were cultured with catechins $(100 \mu \mathrm{M}$ for each) for 1 and $5 \mathrm{hr}$. The fluidity changes of cell membranes were determined by measuring fluorescence polarization with ANS and PNA. Mean \pm S.E. $(n=5) .{ }^{*} p<0.05$ and ${ }^{* *} p<0.01$, compared with control.

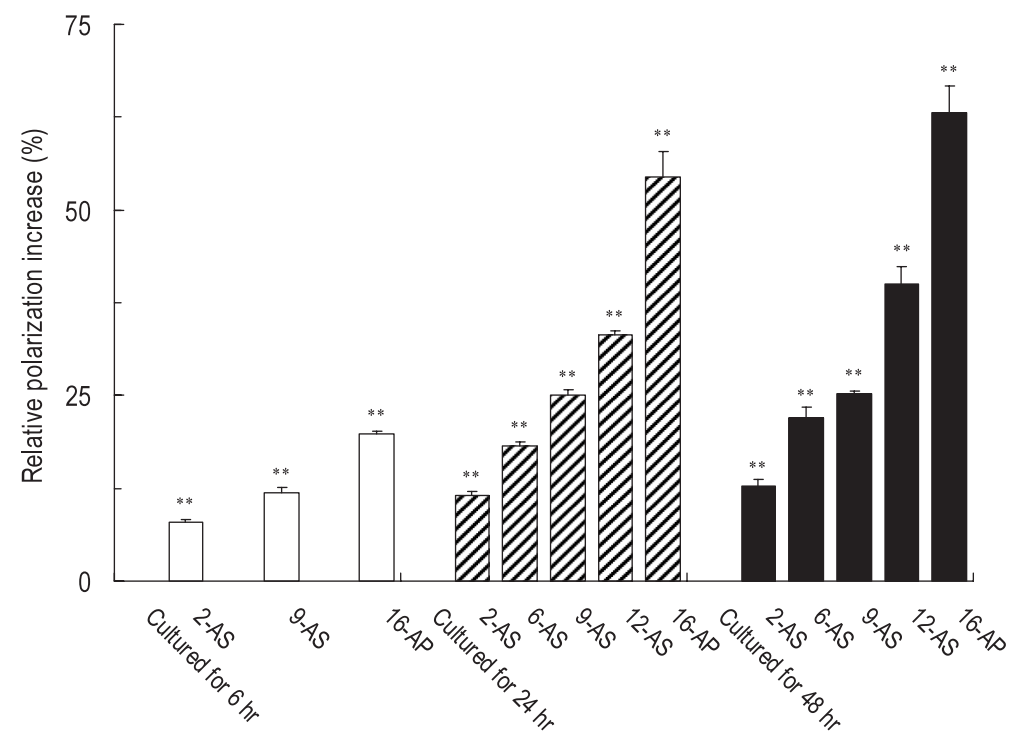

Fig. 2. Effects of EGCG on Different Regions of Cell Membranes

Mouse myeloma cells were cultured with EGCG $(100 \mu \mathrm{M})$ for $6-48 \mathrm{hr}$. The fluidity changes with the gradient extending from the surface to the center of cell membranes were determined by measuring fluorescence polarization with $n$-AS(P). Mean \pm S.E. $(n=5)$. ${ }^{* *} p<0.01$, compared with control.

with the potency being EGCG > ECG by acting on their surfaces and interiors as shown by ANS and PNA polarization increases (Fig. 1). However, nonantiproliferative EC and $\mathrm{C}$ were not or much less effective on tumor cell membranes. The relative potencies to rigidify cell membranes were correlated with those to inhibit cell proliferation, suggesting the relation between antiproliferative and membrane effects of catechins.

For characterizing the cell membrane action, the induced rigidification was compared along graded depths of tumor cell membranes by $n$-AS(P) polarization increases relative to control values. The most antiproliferative EGCG predominantly affected the hydrophobic cores of cell membranes (Fig. 2). Not only its acting site was deeper but also its rigidifying potency was greater with increasing the cell culture time, which is interpreted by the time-dependent penetration into cell membranes.

In order to verify the membrane lipid interaction underlying the effects of catechins on tumor cells, tumor cell model membranes consisting of 


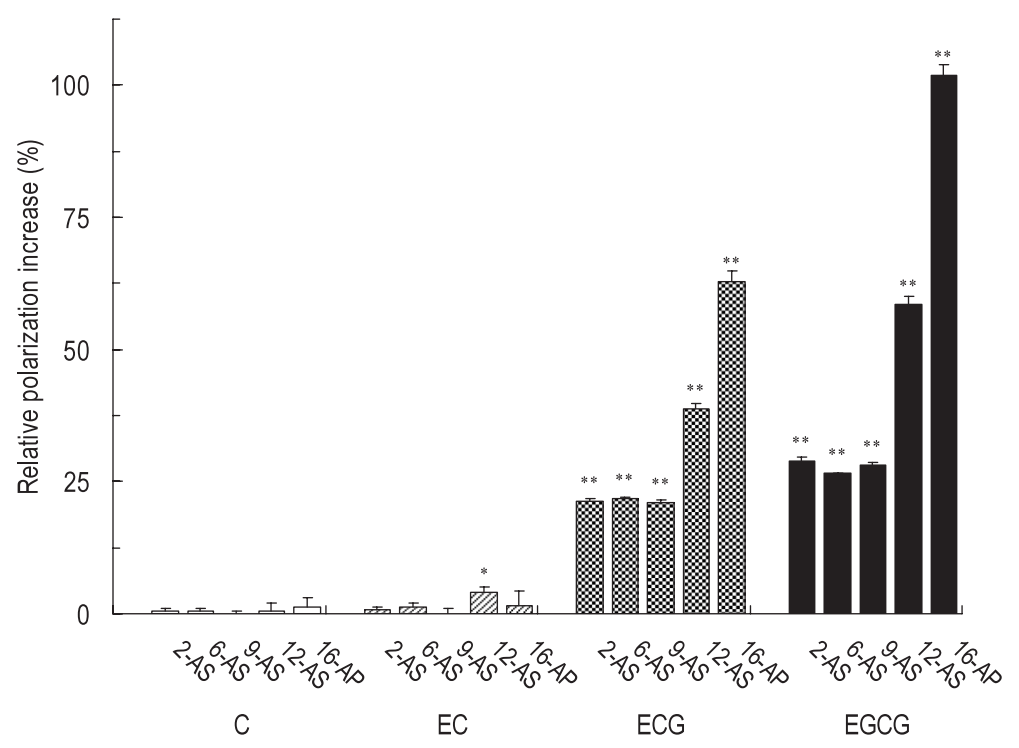

Fig. 3. Interactions of Catechins with Membrane Lipids

Liposomal tumor cell model membranes were treated with catechins $(10 \mu \mathrm{M}$ for each). The fluidity changes with the gradient extending from the surface to the center of lipid bilayers were determined by measuring fluorescence polarization with $n$-AS(P). Mean \pm S.E. $(n=5) .{ }^{*} p<0.05$ and ${ }^{* *} p<0.01$, compared with control.

phospholipids and cholesterol were treated with $\mathrm{C}$, EC, ECG and EGCG. EGCG most intensively rigidified the liposomal membranes at $10 \mu \mathrm{M}$, followed by ECG, EC and C in decreasing order of intensity as shown by $n-\mathrm{AS}(\mathrm{P})$ polarization increases relative to control values (Fig. 3). This rank order agrees with that found in both potencies to rigidify tumor cell membranes and to inhibit the growth of tumor cells. Liposomes and cell membranes also showed the same features (to act on deeper regions) for the membrane effects of catechins. The interactions of catechins with lipid bilayers are responsible for their induced rigidification of tumor cell membranes. The relative polarization increases by EGCG were smaller in cell membranes than in liposomal membranes. Such difference may be due to the binding of EGCG to medium components and membrane proteins during cell culture.

The hydrophobic interaction is related to the membrane effects of catechins. Relative hydrophobicity is estimated to be ECG $>$ EGCG $>$ EC $>$ C based on the reversed-phase chromatographic retention ${ }^{10)}$ and the partition in $n$-octanol/PBS system. ${ }^{18)}$ This rank order suggests the ability of ECG to interact with membrane lipids more intensively than EGCG. ECG was previously reported to be more potent to act on liposomal membranes than EGCG. ${ }^{8,19)}$ In this study, however, the relative membrane activity was reversed between ECG and EGCG. This discrepancy is attributable to the compositional difference of membrane lipids, that is, previous liposomes were prepared with phosphatidylcholine alone, whereas the present ones with different unsaturated phospholipids and cholesterol to resemble tumor cell membranes. Tumor cells change their membrane fluidity by decreasing the cholesterol content and increasing the phospholipids unsaturation degree to elevate proliferative and metastatic activities. ${ }^{4}$ With respect to the role of cholesterol, it is also of interest that EGCG shows the significant bioactivity by selectively interacting with membrane lipid rafts,${ }^{20)}$ in which cholesterol is concentrated to determine the organization, stability and function of membrane microdomains.

Membrane dynamics are so important for cell proliferation that cell cycling is accompanied by membrane fluidity changes and proliferating cells have more fluid membranes than the cells in resting stage. ${ }^{2)}$ The fluidity enhancement of cell membranes and liposomes is counteracted by antitumor drugs. ${ }^{5,6)}$ Membrane-rigidifying catechins inhibit ornithine decarboxylase and cyclooxygenase relevant to cell proliferation and carcinogenesis, ${ }^{21,22)}$ and membrane fluidity-modifiers regulate carcinogen-metabolizing and -activating enzyme activities. ${ }^{7)}$ Fluidity changes also decrease the biochemical reaction efficiency in membrane lipid environments. ${ }^{3)}$ The membrane rigidification by catechins would not only affect cell events relating to proliferation but also depress lipid peroxidation by hindering the diffusion of free radicals in 
lipid bilayers. ${ }^{5,13)}$

In conclusion, the antiproliferative effects of green tea catechins are associated with their interactivities with lipid bilayers to modify cell membrane fluidity, which depend on the structure of catechins and the composition of membrane lipids.

Acknowledgements This study was partly supported by a grant from the San-Ei Gen Foundation for Food Chemical Research (to H. T.).

\section{REFERENCES}

1) Yamamoto, T., Juneja, L. R., Chu, D. -C. and Kim, M. (1997) Chemistry and Applications of Green Tea, CRC Press, Boca Raton.

2) Muller, C. P. and Krueger, G. R. F. (1986) Modulation of membrane proteins by vertical phase separation and membrane lipid fluidity. Basis for a new approach to tumor immunotherapy. Anticancer Res., 6, 1181-1194.

3) Daoud, S. S. (1992) Cell membranes as targets for anti-cancer drug action. Anticancer Drugs, 3, 443453.

4) Shinitzky, M. (1984) Membrane fluidity in malignancy. Adversative and recuperative. Biochim. Biophys. Acta, 738, 251-261.

5) Wiseman, H. (1994) Tamoxifen: new membranemediated mechanisms of action and therapeutic advances. Trends Pharmacol. Sci., 15, 83-89.

6) Yu, J., Cheng, Y., Xie, L. and Zhang, R. (1999) Effects of genistein and daidzein on membrane characteristics of HCT cells. Nutr. Cancer, 33, 100-104.

7) Das, N. P., Shahi, G. S., Moochhala, S. M., Sato, T. and Sunamoto, J. (1992) Effect of 1-methyl-4phenyl-1,2,3,6-tetrahydropyridine (MPTP) and its toxic metabolites on the physicochemical property of the liposomal membrane in relation to their cytochrome P-450 inhibition. Chem. Phys. Lipids, 62, 303-310.

8) Tsuchiya, H. (1999) Effects of green tea catechins on membrane fluidity. Pharmacology, 59, 34-44.

9) Fernández, P. L., Martín, M. J., González, A. G. and Pablos, F. (2000) HPLC determination of catechins and caffeine in tea. Differentiation of green, black and instant teas. Analyst, 125, 421-425.

10) Tsuchiya, H., Sato, M., Kato, H., Okubo, T., Juneja, L. R. and Kim, M. (1997) Simultaneous determination of catechins in human saliva by highperformance liquid chromatography. J. Chromatogr. B, Biomed. Sci. Appl., 703, 253-258.

11) Tsuchiya, H., Nagayama, M., Tanaka, T.,
Furusawa, M., Kashimata, M. and Takeuchi, H. (2002) Membrane-rigidifying effects of anti-cancer dietary factors. Biofactors, 16, 45-56.

12) Furusawa, M., Tsuchiya, H., Nagayama, M., Tanaka, T., Oyama, M., Ito, T., Iinuma, M. and Takeuchi, H. (2006) Cell growth inhibition by membrane-active components in brownish scale of onion. J. Health Sci., 52, 578-584.

13) Arora, A., Byrem, T. M., Nair, M. G. and Strasburg, G. M. (2000) Modulation of liposomal membrane fluidity by flavonoids and isoflavonoids. Arch. Biochem. Biophys., 373, 102-109.

14) Tsuchiya, H., Mizogami, M. and Takakura, K. (2005) Reversed-phase liquid chromatographic retention and membrane activity relationships of local anesthetics. J. Chromatogr. A, 1073, 303-308.

15) Van Blitterswijk, W. J., De Veer, G., Krol, J. H. and Emmelot, P. (1982) Comparative lipid analysis of purified plasma membranes and shed extracellular membrane vesicles from normal murine thymocytes and leukemic GRSL cells. Biochim. Biophys. Acta, 688, 495-504.

16) Valcic, S., Timmermann, B. N., Alberts, D. S., Wächter, G. A., Krutzsch, M., Wymer, J. and Guillén, J. M. (1996) Inhibitory effect of six green tea catechins and caffeine on the growth of four selected human tumor cell lines. Anticancer Drugs, 7, 461-468.

17) Yang, C. S., Chung, J. Y., Yang, G. -Y., Chhabra, S. K. and Lee, M. -J. (2000) Tea and tea polyphenols in cancer prevention. J. Nutr., 130, 472S-478S.

18) Kajiya, K., Kumazawa, S. and Nakayama, T. (2001) Steric effects on interaction of tea catechins with lipid bilayers. Biosci. Biotechnol. Biochem., 65, 2638-2643.

19) Nakayama, T., Hashimoto, T., Kajiya, K. and Kumazawa, S. (2000) Affinity of polyphenols for lipid bilayers. Biofactors, 13, 147-151.

20) Fujimura, Y., Tachibana, H. and Yamada, K. (2004) Lipid raft-associated catechin suppresses the FceRI expression by inhibiting phosphorylation of the extracellular signal-regulated kinase1/2. FEBS Lett., 556, 204-210.

21) Wang, Y. -C. and Bachrach, U. (2002) The specific anti-cancer activity of green tea (-)epigallocatechin-3-gallate (EGCG). Amino Acids, 22, 131-143.

22) Gerhäuser, C., Klimo, K., Heiss, E., Neumann, I., Gamal-Eldeen, A., Knauft, J., Liu, G. -Y., Sitthimonchai, S. and Frank, N. (2003) Mechanismbased in vitro screening of potential cancer chemopreventive agents. Mutat. Res., 523-524, 163-172. 